\title{
M2 Macrophage-Conditioned Medium Inhibits Intervertebral Disc Degeneration in a Tumor Necrosis Factor-a-rich Environment
}

\section{Xiao-Chuan Li}

Gaozhou People's Hospital

Shao-Jian Luo

Gaozhou people's hospital

\section{Wu Fan}

Gaozhou People's Hospital

Tian-Li Zhou

Gaozhou People's Hospital

Chun-Ming Huang

Gaozhou People's Hospital

Maosheng Wang ( $\nabla$ wangmsgzph@163.com )

Gaozhou people's hospital

\section{Research}

Keywords: macrophage, intervertebral disc, M2, inflammation, tissue regeneration

Posted Date: April 22nd, 2021

DOl: https://doi.org/10.21203/rs.3.rs-429415/v1

License: (9) (i) This work is licensed under a Creative Commons Attribution 4.0 International License. Read Full License

Version of Record: A version of this preprint was published at Journal of Orthopaedic Research on February 16th, 2022. See the published version at https://doi.org/10.1002/jor.25292. 


\section{Abstract}

\section{Background}

Inflammation is the primary pathological phenomenon associated with disc degeneration; accordingly, the inflammatory cytokine tumor necrosis factor (TNF-a) plays a crucial role in disc degeneration. M1 macrophages produce proinflammatory cytokines that facilitate the progression of intervertebral disc degeneration (IDD). However, the anti-inflammatory and regenerative effects of M2 macrophages on nucleus pulposus cells (NPCs) in IDD progression remain unknown. Here, we aimed to determine the role of M2 macrophages in IDD progression.

Methods

M2 conditioned medium (M2CM) was harvested and purified from THP-1 cells; it was then used for culturing human NPCs and a mouse intervertebral disc (IVD) organ culture model. NPCs and IVD organ models were divided into the following three groups: group 1 was treated with $10 \%$ fetal bovine serum to actas the control, group 2 was treated with $10 \mathrm{ng} / \mathrm{ml}$ TNF-a, and group 3 was treated with $10 \mathrm{ng} / \mathrm{ml} \mathrm{TNF-}$ $a$ and M2CMto act as the co-culture group. After 3 to 14 days, cell proliferation (CCK-8 assay and western blotting for proliferation markers), extracellular matrix synthesis (quantitative polymerase chain reaction, western blotting, and immunofluorescence), apoptosis (TUNEL staining and western blotting), and NPC senescence (senescence-associated beta-galactosidase staining and western blotting) were assessed.

Results

CD206 and interleukin (IL)-10 levels were increased after $48 \mathrm{~h}$ of induction for M2 macrophages (both $\mathrm{p}<0.01)$. Cell proliferation was decreased in TNF-a-treated NPCs and was inhibited by M2CM co-culture. Moreover, TNF-a treatment enhanced the apoptosis, senescence, and expression of inflammatory factorrelated genes, including IL-6, MMP-13, ADAMTS-4, and ADAMTS-5, whereas M2CM co-culture significantly reversed these effects. M2CM promoted aggrecan and collagen II synthesis but reduced collagen la1 levels in TNF-a treatment groups. Using our established three-dimensional murine IVD organ culture model, M2CM suppressed the inhibitory effect of TNF-a of the TNF-a-rich environment.

Conclusions

Collectively, these results indicate that $\mathrm{M} 2 \mathrm{CM}$ promotes cell proliferation and extracellular matrix synthesis and inhibits inflammation, apoptosis, and NPC senescence. This study therefore highlights the therapeutic potential of M2CM for IDD.

\section{Introduction}

Low back pain is a global issue that affects nearly $80 \%$ of individuals at some time point during their lifetime ${ }^{1}$. Intervertebral disc degeneration (IDD) is considered the pathological basis of low back pain, which reduces the quality of life and generates a massive economic burden ${ }^{2}$. Surgical treatment is a 
widely accepted, ultimate solution for IDD patients with end-stage degeneration, but is associated with the risk of surgical complications, function loss, and poor clinical effects ${ }^{3,4}$. For most patients with earlystage IDD, current therapies are aimed at mitigating symptoms rather than fundamentally treating the pathological condition ${ }^{5}$. Therefore, biological therapy to restore disc morphology and function to inhibit IDD progression is highly significant. The pathophysiology of IDD is characterized by a reduction in the levels of extracellular matrix (ECM) components, including aggrecan and collagen II, and increase in activities of catabolic enzymes, including matrix metalloproteinase-13 (MMP-13) and a disintegrin metalloproteinase with thrombospondin motifs 4 and 5 (ADAMTS- 4 and ADAMTS-5) ${ }^{6}$. Therefore, maintenance of the anabolism and catabolism balance in the disc may prevent or even reverse IDD.

Although the intervertebral disc (IVD) is widely accepted as an immune-privilege organ, immune homeostasis plays an important role in IVD degeneration and regeneration ${ }^{7-9}$. Various types of immune cells such as T and B cells, mast cells, and macrophages have been implicated in IDD ${ }^{10-12}$. Recently, macrophages have garnered attention in this regard with more recognition of the roles of different macrophage phenotypes, including pro-inflammatory $\mathrm{M} 1$, remodeling $\mathrm{M} 2 \mathrm{c}$, and anti-inflammatory $\mathrm{M} 2 \mathrm{a}$ phenotypes, which have been reported in IDD ${ }^{10,13}$. Moreover, studies have reported cellular interactions between M0/M1 macrophages and nucleus pulposus cells (NPCs) under pathological and physiological culture conditions ${ }^{14}$. Furthermore, studies in animal models, including rat, mouse, and dog models, have revealed an increased number of infiltrating macrophages with a high level of inflammatory factors in $I D^{15-17}$. Moreover, a cadaver study demonstrated differences in the distribution of macrophages in different parts of the IVD, with M2 macrophages accumulating more around the granulation tissue, suggesting their remodeling potential in IDD ${ }^{13}$.

Macrophages display plasticity predominantly through the pro-inflammatory state of M1 polarization in the early stage of tissue injury and the anti-inflammation and healing states of M2 polarization in the later stages; the transition state has been described in various tissue injuries ${ }^{18}$. Although studies have increasingly focused on the roles of M0/M1 macrophages and disc cells in IDD, the role of M2 cells remains unclear. M2 macrophages help to maintain tissue homeostasis in the heart ${ }^{19}$, lung ${ }^{20}$, muscle ${ }^{21}$, and spinal cord ${ }^{22}$. We have found that M2 macrophages were also present at high levels in surgical samples of IDD patients, with a significantly increased trend in Pfirrmann grade and age (data not shown). Conditioned medium (CM) contains a mixture of different factors secreted by cells, including growth factors, cytokines, enzymes, nucleic acids, and bioactive lipids, representing a new class of therapeutics with wide applications in disease treatment and injury ${ }^{23}$. M2 macrophage-CM (M2CM) has been reported to inhibit inflammation and apoptosis in chondrocytes, generating a pro-chondrogenic environment by producing immunoregulatory factors ${ }^{24-26}$.

However, it remains unclear whether $\mathrm{M} 2 \mathrm{CM}$ has anti-inflammatory and remodeling potential to alleviate IDD progression. IDD is primarily associated with inflammation; thus, the pro-inflammatorycytokine tumor necrosis factor-alpha (TNF-a) has been widely used to mimic the degeneration conditions in IDD $27-29$. Accordingly, we hypothesized that M2 macrophages attenuate the harmful effects of TNF-a, including the 
effects on the proliferation, senescence, apoptosis, and inflammation of NPCs. To test this possibility and provide a foundation for the potential therapeutic applications of M2CM in IDD, the aim of this study was to investigate the anti-inflammatory, anti-apoptotic, and anti-senescence effects of M2CM in TNF-atreated NPCs.

\section{Materials And Methods}

\subsection{Ethics statement}

The study was performed according to the amended declaration of Helsinki; human NP tissues were isolated from eight patients and the detailed information regarding the patients is presented in Table 1. In addition, for IVD culture models, 2-month-old male C57 mouse were used in this study. The experiment was performed according to the amended declaration of Helsinki and was approved by the Committee of Gaozhou People's Hospital (No. 2018-012). Informed consent was obtained from each patient.

\subsection{Human NPisolationand human NPC culture}

NPCs were isolated and harvested as previously reported ${ }^{30}$. Briefly, NP samples were collected and immediately transported to a cell culture room under sterile conditions. After washing with phosphatebuffered saline (PBS) three times, the NP tissues were cut into small pieces $\left(<1 \mathrm{~mm}^{3}\right)$ and digested with $0.2 \%$ collagenase II (Sigma, USA) for $3-4 \mathrm{~h}$ at $37^{\circ} \mathrm{C}$ in a humidified incubator. The suspended cells were then passed through a 200- $\mu \mathrm{m}$ mesh filter and centrifuged at $150 \times g$ for $5 \mathrm{~min}$; this was followed by two washes with PBS. Finally, the cells were cultured in culture medium, consisting of F12 DMEM (HyClone), $10 \%$ fetal calf serum (Gibco), and $1 \%$ penicillin/streptomycin (Gibco) in $25-\mathrm{cm}^{2}$ cellcultureflasks at a density of $1 \times 10^{5}$ cells $/ \mathrm{mL}$ in a humidified incubator at $37^{\circ} \mathrm{C}$ under $5 \% \mathrm{CO}_{2}$. After 3 days, the suspended cells and medium were removed, and the adherent cells were cultured and the medium was replaced every $2-3$ days. As the cells reached $70 \%-80 \%$ confluency, the primary cells were harvested and passaged. Passage 1 (P1) NPCs were harvested with $0.25 \%$ trypsin-ethylenediaminetetraacetic acid (EDTA; Sigma) for $1 \mathrm{~min}$ and subcultured at a ratio of 1:3. After the cells were gradually passaged, P3 cells were harvested or cryopreserved for further experiments.

\subsection{Isolation and cultureofIVD organ culture models}

Eight-week old male C57 mice were obtained from experimental animal center of Southern Medical University. The mice were euthanized, and then the tails were cut from the base segment of the tail. After soaking in lodophor for $5 \mathrm{~min}$, the tail skin was removed and tail spines were harvested under sterile condition. The Co7/8 andCo8/9IVD were harvested under sterile conditions, and the dissected IVDs were rinsed in sterile PBS and immediately placed in culture medium as mouse IVD organmodels. Every five IVDs were cultured in $25-\mathrm{cm}^{2}$ cell culture bottles with $15 \mathrm{~mL}$ culture medium containingF12 DMEM (HyClone), 10\% fetal calf serum (Gibco), and 1\% penicillin/streptomycin (Gibco). Samples were cultured in a humidified incubator at $37^{\circ} \mathrm{C}$ under $5 \% \mathrm{CO}_{2}$, and the culture medium was replaced every 3 days. 


\subsection{Harvest of human M2CM and treatment of samples}

The THP-1cells were seeded at a density of $2 \times 10^{6}$ in $175-\mathrm{cm}^{2}$ cell culture bottle for $24 \mathrm{~h}$, and then treated with PMA (100 ng/mL; RD, Littleton, CO) for 24h. Thereafter, they were changed into serum-free media and treated with or without IL-4 (100 ng/mL; RD) for another $24 \mathrm{~h}$. The supernatant medium was replaced with serum-free medium and cultured for an additional $24 \mathrm{~h}$. Finally, the corresponding supernatant conditioned medium was obtained and centrifuged for $15 \mathrm{~min}$ at $4^{\circ} \mathrm{C}$ at $600 \times \mathrm{g}$ to remove cellular debris and repeated at $1500 \times \mathrm{g}$. The harvested supernatant conditioned medium was defined as M2 macrophage-conditioned medium (M2CM) in the study (Figure 1A). After culturing NPCs or IVD organ models in medium for $24 \mathrm{~h}$, they were divided into three groups as follows: group 1 was treated with $10 \%$ FBS culture medium as the control, group 2 was treated with $10 \mathrm{ng} / \mathrm{mL}$ TNF-a, and group 3 was treated with $10 \mathrm{ng} / \mathrm{mL}$ TNF-a and 30\% M2CM as the co-culture group. The entire procedure is shown in Figure $1 \mathrm{~A}$.

\subsection{Cell proliferation assay}

Cell Counting Kit-8 (CCK-8; Dojindo Laboratories, Japan) was used as previously described to measure cell proliferation ${ }^{28}$. Briefly, NPCs were seeded in 96 -well plates $\left(2 \times 10^{3}\right.$ cells/well) and different groups were incubated for 24,48 , and $96 \mathrm{~h}$. After removing the culture medium and M2CM, $10 \mu \mathrm{L}$ of CCK-8 solution was added to $100 \mu \mathrm{L}$ of fresh medium and the mixture was incubated at $37^{\circ} \mathrm{C}$ for $1 \mathrm{~h}$. Finally, the samples were added to 96 -well plates for final measurements. The absorbance of the solution was measured at $450 \mathrm{~nm}$ using a microplate absorbance reader (Bio-Rad, USA). A blank 96-well plate was used for zero setting. All experiments were performed four times for each sample.

\subsection{Cell apoptotic ratedetermination using the TUNEL assay}

Cell apoptosis was confirmed using the TUNEL assay (Promega, Madison, WI) according to the manufacturer's instructions and previous reports ${ }^{28}$. Briefly, the cells were washed with PBS three times and fixed in $4 \%$ paraformaldehyde for $30 \mathrm{~min}$. Thereafter, the cells were washed with PBS three times and incubated with TdT-mediated dUTP for $1 \mathrm{~h}$ in the dark. The cells with the entire nuclear area labeled redwere defined as apoptotic cells. The cell nuclei were stained with DAPI solution (1:1000; Invitrogen) for $2 \mathrm{~min}$ at room temperature in the dark. The cells were counted and averaged for three different sets of experiments. The positivity of cell apoptosis was analyzed using Image-Pro Plus software (Version 5.1; Media Cybernetics, Inc., USA).All experiments were performed four times for each sample.

\subsection{Senescence-associated $\beta$-galactosidase (SA- $\beta$-gal) staining}

After 5 days of incubation, NPCs were analyzed using a Senescence $\beta$-Galactosidase Staining Kit (Beyotime Institute of Biotechnology). Briefly, the cells were washed with PBS, fixed in the SA- $\beta$-gal fixative solution for $15 \mathrm{~min}$ atroom temperature, rinsed three times with PBS, and then incubated in SA- $\beta$ gal working solution (Reagents $\mathrm{A}, \mathrm{B}, \mathrm{C}$, and $\mathrm{X}-\mathrm{Gal}$ ) overnight at $37^{\circ} \mathrm{C}$ under atmospheric conditions. 
Quantification was performed by counting the number of SA- $\beta$-gal-positive cells and thetotal number of cells from three randomly selected areas for each sample. All analyses were carried out in triplicate.

\subsection{Immunofluorescence microscopy}

NPCs were plated in flat-bottomed 24-well plates $\left(1 \times 10^{4} /\right.$ well $)$ and fixed with $4 \%$ paraformaldehyde for 30 min, permeabilized with $0.2 \%$ triton X-100 in PBS (PBS-T) for 10 min, blocked with PBS containing $5 \%$ FBS, and incubated with antibodies against collagen II (1:100; Abcam, UK) at $4^{\circ} \mathrm{C}$ overnight. As a negative control, the cells were incubated with antibody diluents without antibodies under similar conditions. After washing, the cells were incubated with anti-rabbit secondary antibody (Jackson, USA) at a dilution of 1:100 for $1 \mathrm{~h}$ at room temperature. Following this, the cells were washed three times and the cell nuclei were stained with DAPI solution (1:1000; Invitrogen) for 2 min at room temperature. The samples were examined and photographed using a fluorescence microscope (FV-1000; Olympus). For quantitative examination, the immunostaining results for the cells were analyzed using Image-Pro Plus software (Version 5.1; Media Cybernetics, Inc.).

\subsection{Real-time PCR analysis}

After incubation under different conditions for 2days, the total RNA was extracted from NPCs or IVDs, using TRIzol (Invitrogen) according to the manufacturer's instructions. The RNA concentration was determined by spectrophotometry and RNA was reverse-transcribed using the PrimeScript ${ }^{\mathrm{T}}$ RT Master Mix (TaKaRa, China). qPCR was performed in triplicate in 96-well plates, using the SYBR Premix Ex Taq $\mathrm{Kit}$; the final volume of the reaction mixture was $20 \mu \mathrm{L}$. All primers were obtained from Sangon (Shanghai, China) and are listed in Supplementary Table 1. qPCR was performed using the One Step SYBR* PrimeScript RT-PCR Kit (TaKaRa, China). GAPDHwas used for normalization. The cycle threshold values were obtained and data were normalized to GAPDH expression using the $2^{-\triangle \Delta \mathrm{Ct}}$ method.

\subsection{Total protein isolation and western blotting}

Western blotting was performed as previously described ${ }^{32}$. Briefly, the total proteins were extracted from cells with $1 \times$ radioimmunoprecipitation assay (RIPA) buffer containing protease and phosphatase inhibitors (Roche, Mannheim, Germany). The concentration of total proteins was determined using the Pierce Bicinchoninic Acid (BCA) Protein Assay kit (Thermo Fisher Scientific, Rockford, IL) following the manufacturer's instruction. Subsequently, $30 \mu \mathrm{g}$ of protein samples was separated by sodium dodecyl sulfate-polyacrylamide gel electrophoresis (SDS-PAGE) and then transferred onto polyvinylidene fluoride (PVDF) membranes (Perkin Elmer, Boston, MA). After being blocked with $1 \times$ Tris-buffered saline and Tween 20 (TBST) containing 5\% blotting-grade blocker nonfat dry milk (Bio-Rad, Hercules, CA), the membranes were incubated overnight with appropriate primary antibodies at $4^{\circ} \mathrm{C}$, followed by incubation with the horseradish peroxidase (HRP)-conjugated secondary antibody (Cell Signaling Technology, Beverly, MA; 1:1,000). Subsequently, the protein bands on the blots were visualized using the Clarity Western ECL Kit (Bio-Rad), and imaged using the ChemiDoc Touch Imaging System (Bio-Rad). The primary antibodies against Sox9 (82630), BAX(2774), Bcl-2(4223), PCNA(13110), cyclin D1 (55506), and 
$\beta$-tubulin (2146) were purchased from Cell Signaling Technology (Beverly, Massachusetts), and all used at 1:1,000 dilution. The primary antibodies against Collagen I (ab138492, 1:1,000), and Aggrecan (ab3773, 1:100) were from Abcam (Cambridge, Massachusetts). Quantification by densitometry was performed using Image-Pro Plus software (Version 5.1; Media Cybernetics, Inc.).

\subsection{Statistical analysis}

The results are shown as mean \pm standard deviation(SD).Student's $t$-test or analysis of variance (ANOVA)was used to determine the statistical difference between groups. Mann-Whitney non-parametric test was performed to compare mean positivity between groups.All statistical analyses were carried out using SPSS software (V11.0; SPSS, Inc., Chicago, IL). Differences were considered statistically significant at $\mathrm{P}<0.05$. All quantitative results were calculated from a minimum of three biological replicates.

\section{Results}

\subsection{Identification of polarized M2 macrophages}

THP-1 cells were cultured in a cell culture bottle in suspension medium and treated with PMA for $24 \mathrm{~h}$; the cells gradually adhered to the bottom of the culture bottle as M0 macrophages (Figure 1B). Thereafter, the cells differentiated into M2 macrophages in the presence of IL-4 for another $24 \mathrm{~h}$ in serum-free medium, exhibiting a homogeneous elongated spindle-like morphologywith slender tentacles (Figure 1B). The expression of M2 macrophage markers CD206 and IL-10 was significantly upregulated in M2induced macrophages compared with that in $\mathrm{M0}$ macrophages (both $p<0.01$, Figure $1 \mathrm{C}$ and $\mathrm{D}$ ).

\subsection{M2CM promoted the proliferation of TNF-a-treated NPCs}

The CCK-8 assay was performed to evaluate the proliferative potential of NPCs. The optical density (OD) of TNF-a-treated NPCs was significantly lower than that of the control group at 24,48 , and $96 \mathrm{~h}$ (all p $<0.05$; Figure 2A). Interestingly, in the TNF-a-treated groups, co-culture with M2CM resulted in a higher OD than that observed in cells treated with only TNF-a (all $p<0.05$; Fig. $2 \mathrm{~A}$ ). Additionally, a significantly lower number of NPCs was detected in the co-culture group than in the TNF-a-treated groups at 24, 48, and $96 \mathrm{~h}$ ( $p<0.05, p<0.05$, and $p<0.01$, respectively; Figure 2B). In contrast, in TNF-a-treated NPCs, a higher cell number was observed in the M2CM co-culture group, with a significant difference at all time points (all $p$ $<0.05$; Figure 2B). Furthermore, western blotting for the cell proliferation markers PCNA and cyclinD1 in the control group and TNF-a-treated NPCs with or without M2CM co-culture showed significant downregulation in the expression of both markers in TNF-a-treated NPCs $(p<0.01$ and $p<0.05)$ compared with that in the control group (Figure 2C); however, co-culture of TNF-a-treated NPCs with M2CM upregulated the expression of PCNA and cyclinD1 (both $p<0.05$; Figure 2D and E).

\subsection{M2CM protected TNF-a-treated NPCs from apoptosis}

The effect of M2CM on TNF-a-induced apoptosis of NPCs was assessed using the TUNEL assay. Upon treatment with TNF-a for 3days, the apoptotic rate gradually increased in NPCs compared with that in the 
control group $(p<0.001)$, whereas co-culture with M2CM significantly mitigated this effect $(p<0.01$; Figure 3A, B). As shown in Figure 3C-E, western blotting revealed that the expression of the pro-apoptotic protein Bax was upregulated, whereas the expression of the anti-apoptotic protein Bcl-2 was downregulated in TNF-a-treated NPCs $(p<0.01$ and $p<0.05$, respectively); however, these effects were reversed upon coculturing with $\mathrm{M} 2 \mathrm{CM}$ (both $p<0.05$ ).

\subsection{M2CM upregulated ECM components in TNF-a-treated NPCs}

To evaluate the effect of M2CM on ECM biosynthesis, immunofluorescence staining of collagen II in NPCs was performed (Figure 4A). After treatment with TNF-a for 3days, the OD value significantly decreased $(p<0.01$; Figure 4B); however, this effect was considerably inhibited in the co-culture group ( $p$ $<0.05$; Figure 4B). Western blotting was performed to analyze the changes in aggrecan and collagen I expression (Figure 4C). Aggrecan expression was also downregulated in the TNF-a-treated group $(p<0.05$; Figure 4D) but was upregulated in the co-culture group ( $p<0.05$; Figure 4D), whereas collagen I expression displayed the reverse pattern (both $p<0.05$; Figure $4 \mathrm{E}$ ). Finally, we investigated ECM-related gene expression by evaluating the expression of genes encoding collagen type la1, collagen type lla1, and aggrecan. Although the expression of ECM-related genes (aggrecan and collagen Ila1) was downregulated in TNF-a-treated NPCs ( $p<0.001$ and $p<0.01$, respectively), M2CM significantly eliminated this inhibitory effect (both $p<0.05$; Figure $4 \mathbf{F}, \mathbf{G}$ ). Conversely, the expression of collagen type la1 was further upregulated in the TNF-a-treated group $(p<0.05$; Figure $4 \mathrm{H})$; co-culturing with M2CM significantly inhibited this increase $(p<0.05$; Figure $4 \mathrm{H})$. Together, these results indicated that M2CM potentially promotes matrix synthesis in TNF-a-treated NPCs.

\subsection{M2CM decreasedsenescence and inflammatory factor levels in TNF-a-treated NPCs}

Cell senescence was analyzed via SA- $\beta$-gal staining and determination of senescence-associated gene expression. The number of SA- $\beta$-gal-positive NPCs increased upon TNF-a treatment compared with that in the control group after 7 days ( $p<0.01$; Figure 5 A and B). However, when co-culturing TNF-a-treated cells with M2CM, the number of SA-B-gal-positive cells significantly decreased ( $p<0.05$; Figure $5 \mathrm{~A}$ and $\mathbf{B})$. With respect to cell senescence-related genes, TNF-a significantly upregulated the expression of p16, p21, and p53 compared with that in the control group $(p<0.01, p<0.01$, and $p<0.05$, respectively; Figure 5CE);however, M2CM downregulated the expression of these markers after TNF-a treatment (all $p<0.05$; Figure 5C-E). The effect of TNF- $a$ on the secretion of inflammatory factors (IL-6) and extracellular proteases (MMP-13, ADAMTS-4, and ADAMTS-5) by NPCs was assessed after treatment for 48h. TNF-a significantly upregulated the expression of all four inflammatory genes compared with that in the control group ( $p<0.05, p<0.001, p<0.01$, and $p<0.001$, respectively; Figure 5F-1); however, M2CM significantly downregulated these markers after TNF-a treatment (all $p<0.05$; Figure 5F-I).

\subsection{M2CM decreased IVD degeneration in TNF-a-treated IVD organ cultures}

To evaluate the effect of M2CM on ECM biosynthesis, HE staining of mouse IVD organ models was performed. After 2 weeks of culture, the nucleus pulposus (NP)tissue in the control group displayed a 
spherical morphology and accounted for $50 \%$ of the disc area, and NPCs displayed a stellar or spherical morphology. In contrast, in the TNF-a-treated group, the NP constituted $25 \%-50 \%$ of the disc area, and large, spherical NPCs were separated by dense areas of a proteoglycan matrix (Figure 6A). However, these degeneration effectswere reversed in both NP and NPCs (Figure 6A). We further analyzed the annulus fibrosus (AF) and annulus fibrosus cells (AFCs) and found thatthe ruptured or serpentine fibers constituted $<25 \%$ of the AF. Furthermore, fibroblasts, comprising $>75 \%-90 \%$ of cells, were detected in the control group, whereas the ruptured or serpentine fibers constituted $>50 \%$ of the AF. Moreover, chondrocytes, comprising $>75 \%$ of the cells, were detected in the TNF-a-treated group (Figure $6 \mathrm{~A}$ ). Notably, with co-culture in M2CM, the degeneration of both AF and AFCs was inhibited (Figure 6A). Finally, the border between the NP and AF resulted from minimal, moderate, and severe interruption in the control, co-culture, and TNF-a-treated groups, respectively (Figure 6A). The modified histological grading system of IVD was used for further quantitative analysis, and the results demonstrated that the TNF-atreated group displayed significant degeneration-related morphologicalchanges with higher scores than the control group ( $p<0.05$, Figure 6B), whereas the co-culture group displayed a significant decreasewithlower scores ( $p<0.05$, Figure $6 \mathrm{~B}$ ). Furthermore, we investigated ECM synthesis by evaluating the expression of genes encoding collagen type lla1 and aggrecan. Although the expression of ECM proteins (aggrecan and collagen Ila1) was decreased in the TNF-a-treated mouse IVD model (both $p$ $<0.01$, Figure 6C and D), M2CM significantly abolished this inhibitory effect (both $p<0.05$, Figure $6 \mathrm{C}$ and D).

\section{Discussion}

M2 macrophages can downregulate pro-inflammatory cytokines and promote tissue remodeling by producing anti-inflammatory cytokines, including transforming growth factor beta (TGF- $\beta$ ), vascular endothelial growth factor, IL-10, and insulin-like growth factor $18,33,34$. Furthermore, M2CM has the potential to regenerate numerous tissues; it exerts different effects such as promoting retinal neovascularization, odontogenic differentiation, and epithelial-mesenchymal transition. Importantly, in cartilage cells, which are close to the intervertebral disc cells, macrophages acting as immune cells are of importance in the symptomology and structural progression of osteoarthritis ${ }^{33,35,36}$. However, whether it exerts similar effects in NPCs during IDD progression has remained unknown. To our knowledge, no study has focused on the protective effect of M2CM in IDD to date. Hence, consistent with other studies, we mimicked the inflammatory environment using TNF-a to promote apoptosis, cell senescence, and ECM catabolism in NPCs. Thereafter, we compared these effects in cells with or without TNF-a treatment cultured in M2CM. Together, our results show that the protective effects of M2CM in IDD were mediated through the promotion of cell proliferation; synthesis of ECM; and inhibition of inflammation, apoptosis, and NPC senescence, thereby alleviating IDD. Our results provide fundamental evidence regarding the clinical application of M2CM therapies.

Macrophage reprogramming, characterized by the transformation of M1 to M2 macrophages, is reportedly an effective treatment alternative for osteoarthritis ${ }^{18,36}$. In cartilage degeneration, the pro- 
inflammatory M1 macrophages contribute to osteoarthritis, whereas M2 macrophages can reverse this effect or favor chondrogenesis. In IDD, a mixed population of M1 and M2 macrophages was observed in both patients and mouse models, suggesting that both cell types contribute to IDD pathophysiology ${ }^{13,37}$. Recent studies have reported interactions between macrophages and IVD cells ${ }^{14,38}$. Although M2 macrophages are known as wound-healing and remodeling macrophages, and provide a protective environment in injured tissues, their potential anti-inflammatory effects and ability to mediate remodeling in IDD have remained unknown. In this study, we successfully induced the differentiation of THP-1 cells to $\mathrm{M} 2$ macrophages, as previously described ${ }^{14,32}$. The change in cell morphology and significant upregulation of the M2 surface markers CD206 and IL-10 indicated that polarization was successful. In the conditioning medium, we used $30 \% \mathrm{M} 2 \mathrm{CM}$ in the total culture medium in accordance with a previously reported similar co-culture system ${ }^{14,36}$. Thus, we successfully induced the generation of M2 macrophages and harvested M2CM by supplementing IL-4 to THP-1 cells.

Disc degeneration results in a concomitant reduction in the number of NPCs, which are responsible for the production of NP matrix components. Therefore, regenerative therapies depending on the stimulation of cell proliferation are promising and feasible. NPC proliferation decreased in the TNF-a-treated group; however, proliferation was restored upon $\mathrm{M} 2 \mathrm{CM}$ co-culture. Furthermore, the increase in cell number was confirmed to be due to an increase in cell proliferation rather than the inhibition of cell death. These data are critical for the development of potential treatments aimed at inhibiting the reduction in cell number observed during IDD ${ }^{39}$. This pro-proliferative effect can be potentially attributed to several cytokines, including TGF- $\beta 1$, ARG-1, and chemokine ligand 18 (CCL-18), which reportedly promote cell proliferation $24,40,41$. However, further studies are still needed to determine the detailed mechanism underlying the observed effects.

Another important finding of this study is that M2CM decreased the incidence of NPC apoptosis. Excessive disc cell apoptosis, leading to decreased cell density and ECM catabolism, exerts a detrimental effect in IDD ${ }^{42,43}$. Here, the TUNEL assay showed that the increased apoptotic rate of NPCs treated with TNF-a was significantly reversed upon co-culturing with M2CM. Furthermore, the expression of the proapoptotic protein Bax and the anti-apoptotic protein Bcl-2 was significantly upregulated and downregulated, respectively, in TNF-a-treated NPCs, and these changes were significantly inhibited upon co-culturing with M2CM. Notably, aberrant apoptosis of NPCs is considered a major cellular phenomenon associated with IDD ${ }^{44}$. Together, these results indicate that the apoptotic rate of TNF-a-treated NPCs was reduced upon co-culturing with $\mathrm{M} 2 \mathrm{CM}$.

The balance between ECM anabolism and catabolism by disc cells is disrupted by pro-inflammatory cytokines during IDD ${ }^{45}$. Hence, inhibition of these inflammatory cytokine-mediated pathological processes might promote NPC synthesis and result in increased ECM deposition. In this study, we used exogenous TNF-a stimulation to mimic the degeneration environment, and we found that M2CM increases ECM synthesis by promoting both gene and protein expression of aggrecan and collagen lla1. However, the downregulation of collagen la1 expression suggests that the inhibitory effect of $\mathrm{M} 2 \mathrm{CM}$ 
during fibrosis further facilitates IVD regeneration. Together, these results indicate that M2CM promotes ECM synthesis in TNF-a-treated NPCs. Several cytokines such as TGF- $\beta 1$, ARG-1, and CCL-18 have been reported in $\mathrm{M} 2 \mathrm{CM}$ with pro-chondrogenic effects ${ }^{18}$. Hence, we speculate that these factors may be key regulators in the M2CM responsible for protecting TNF-a-treated NPCs in our study. Nonetheless, further studies are required to isolate and identify these factors for clinical application.

NPCs maintain normal NP tissue homeostasis ${ }^{46}$. However, the gradual increase in NPC senescence during IDD has a detrimental effect by decreasing the number of functional cells; thus, the inhibition of NPC senescence is considered an important strategy for IDD treatment. SA- $\beta$-gal is a senescent cell biomarker ${ }^{47}$. In our study, a significantly lower percentage of SA- $\beta$-gal-positive cells were observed in the co-culture group than in the TNF-a group, indicating a protective role of M2CM on NPC senescence. Our results suggest that $\mathrm{M} 2 \mathrm{CM}$ can attenuate premature senescence of NPCs in an inflammatory microenvironment. The telomere-based p53-p21-pRb pathway and the stress-based p16-pRb pathway are the predominant pathways in IDD ${ }^{44}$. Our results suggest that both pathways mediate the effect of TNF-a on NPCs in vitro, and that the extrinsic factor p16 plays a particularly crucial role, as indicated by its marked upregulation. Moreover, co-culture with M2CM led to the downregulation of pro-inflammatory cytokines (IL-6) and extracellular proteases (MMP-13, ADAMTS-4, and ADAMTS-5), which are widely accepted as risk factors during disc degeneration. Together, these findings further strengthen our conclusion.

Finally, three-dimensional organ culture models for IVD have been widely used to investigate the effects of specific treatment strategies in a controlled environment ${ }^{48}$. Coccygeal animal disc models are most commonly used owing to their availability and similarity to human IVDs, and the mouse model is highly similar to the human lumbar disc with respect tothe disc height, anteroposterior width, NP, disc torsion mechanics, axial compression mechanics, and glycosaminoglycan content ${ }^{49}$. Herein, the mouse IVD organ culture model was used to assess the biological effect of M2CM in TNF-a-added medium. A modified histological scoring system was used to analyze the protective effect of $\mathrm{M} 2 \mathrm{CM}^{50}$. We found that treatment with only TNF-a significantly increased the score in the IVD organ culture model, whereas co-culturing with $\mathrm{M} 2 \mathrm{CM}$ inhibited this effect. Furthermore, our results indicate that degenerative culture conditions considerably attenuated the downregulation of collagen Ila and ACAN expression, thus demonstrating the regeneration potential of $\mathrm{M} 2 \mathrm{CM}$ in the three-dimensional model.

Although the present results highlight the potential of $\mathrm{M} 2 \mathrm{CM}$ in disc repair therapy, this study also has some limitations. First, the degenerated environment in IDD is multifactorial and difficult to mimic completely with only exogenous TNF-a supplementation. Second, the microenvironment regulated by proand anti-inflammatory macrophages is usually in a dynamic balance, and M1/M2 is associated with the two major and opposing activities of macrophages ${ }^{51}$. Hence, the predominant role of M2 cells likely involvesinhibiting or maintaining a balance with M1 macrophages. Therefore, it is difficult to analyze this potential, which may limit the applicability of our results to some extent. Furthermore, although the present conclusion is based on evidence obtained from invitro and IVD culture models, the therapeutic 
effects on disc degeneration in vivo still need to be assessed in an animal model. Furthermore, the precise cytokines and mechanism of action of $\mathrm{M} 2 \mathrm{CM}$ remain unclear and warrant further investigation; our future work will focus on elucidating the underlying mechanisms. Finally, the sources of NPCs from disc cells of different species may interfere with M2CMto some extent, as the primary NPCs originate from different degenerating IVD samples.

\section{Conclusion}

TNF-a successfully induced premature senescence of NPCs and exerted detrimental effects, including the proliferation and senescence of NPCs, and the expression of matrix macromolecules in NPCs. Our study revealed that M2CM positively influences NPCs and IVD culture models in an anti-inflammatory microenvironment by increasing cell proliferation, decreasing cellular senescenceand apoptosis, and promoting ECM accumulation. Our study provides novel insights into the therapeutic potential of $\mathrm{M} 2 \mathrm{CM}$ in IDD. Further studies are warranted to provide adequate evidence regarding the therapeutic potential of resident IVD cells producing sufficient ECM for IDD treatment.

\section{Abbreviations}

IDD, intervertebral disc degeneration; IVD, intervertebral disc; M2CM, M2-conditioned medium; NPC, nucleus pulposus cell

\section{Declarations}

\section{Acknowledgments}

We thank Dan-qin Tan and Jin-Mei Liao from the Department of Orthopedics, Gaozhou People's Hospital, for their valuable assistance in obtaining human NP specimens.

\section{Funding}

This work was supported by the National Natural Science Foundation of China (Grant No 81802130), China Postdoctoral Science Foundation (2018M630968), and Natural Science Foundation of Guangdong Province (2018A030310462).

\section{Conflict of Interest}

The authors have stated explicitly that there are no conflicts of interest in connection with this article.

\section{Author Contributions}

LXC, WMS, and HCM conceived and designed the experiments. LXC, LSJ, ZTL, CW, and FW performed and analyzed the data, interpreted the data, and wrote the manuscript. LXC and WMS provided reagents and reviewed the manuscript for intellectual content. 
Consent for publicationNot applicable.

\section{Availability of Data and Materials}

The details of information used and analyzed for the current study are available from the corresponding author on reasonable request.

\section{Ethics approval and consent to participate}

The study was performed according to the amended declaration of Helsinki; human NP tissues were isolated from eight patients and the detailed information regarding the patients is presented in Table 1. In addition, for IVD culture models, 2-month-old male C57 mouse were used in this study. The experiment was performed according to the amended declaration of Helsinki and was approved by the Committee of Gaozhou People's Hospital (No. 2018-012). Informed consent was obtained from each patient.

\section{References}

1. Deyo RA, Mirza SK. Clinical practice. Herniated lumbar intervertebral disk. N Engl J Med. (2016) 18:1717-1772.

2. Vos T, Abajobir AA, Abate KH, Abbafati C, Abbas KM, Abd-Allah F, et al. Global, regional, and national incidence, prevalence, and years lived with disability for 328 diseases and injuries for 195 countries, 1990-2016: A systematic analysis for the global burden of disease study 2016. Lancet. (2017) 390:1212-1259.

3. Bailey CS, Rasoulinejad P, Taylor D, Sequeira K, Miller T, Watson J, et al. Surgery versus conservative care for persistent sciatica lasting 4 to 12 months. N Engl J Med. (2020) 12:1093-1102.

4. Ramaswami R, Ghogawala Z, Weinstein JN. Management of sciatica. N Engl J Med. (2017) 12:11751177.

5. Desai S, Jayasuriya CT. Implementation of endogenous and exogenous mesenchymal progenitor cells for skeletal tissue regeneration and repair. Bioengineering (Basel). (2020) 3.86.

6. Risbud MV, Shapiro IM. Role of cytokines in intervertebral disc degeneration: Pain and disc content. Nat Rev Rheumatol. (2014) 1:44-56.

7. Liu ZH, Sun Z, Wang HQ, Ge J, Jiang TS, Chen YF, et al. Fasl expression on human nucleus pulposus cells contributes to the immune privilege of intervertebral disc by interacting with immunocytes. Int $J$ Med Sci. (2013) 8:1053-1060.

8. Sun Z, Liu B, Luo ZJ. The immune privilege of the intervertebral disc: Implications for intervertebral disc degeneration treatment. Int J Med Sci. (2020) 5:685-692.

9. Cunha C, G QT, Ribeiro-Machado C, C LP, Ferreira JR, Molinos M, et al. Modulation of the in vivo inflammatory response by pro- versus anti-inflammatory intervertebral disc treatments. International journal of molecular sciences. (2020) 5: 1730. 
10. Yang C, Cao P, Gao Y, Wu M, Lin Y, Tian Y, et al. Differential expression of p38mapk alpha, beta, gamma, delta isoforms in nucleus pulposus modulates macrophage polarization in intervertebral disc degeneration. Sci Rep. (2016) 6:22182.

11. Wiet MG, Piscioneri A, Khan SN, Ballinger MN, Hoyland JA, Purmessur D. Mast cell-intervertebral disc cell interactions regulate inflammation, catabolism and angiogenesis in discogenic back pain. Sci Rep. (2017) 7:12492.

12. Ohba T, Haro H, Ando T, Koyama K, Hatsushika K, Suenaga F, et al. A potential role of thymic stromal lymphopoietin in the recruitment of macrophages to mouse intervertebral disc cells via monocyte chemotactic protein 1 induction: Implications for herniated discs. Arthritis Rheum. (2008) 11:35103519.

13. Nakazawa KR, Walter BA, Laudier DM, Krishnamoorthy D, Mosley GE, Spiller KL, et al. Accumulation and localization of macrophage phenotypes with human intervertebral disc degeneration. Spine J. (2018) 2:343-356.

14. Yang $H$, Liu B, Liu Y, He D, Xing Y, An Y, et al. Secreted factors from intervertebral disc cells and infiltrating macrophages promote degenerated intervertebral disc catabolism. Spine (Phila Pa 1976). (2019) 9:E520-529.

15. Miyagi M, Uchida K, Takano S, Fujimaki H, Aikawa J, Sekiguchi H, et al. Macrophage-derived inflammatory cytokines regulate growth factors and pain-related molecules in mice with intervertebral disc injury. J Orthop Res. 2018.

16. Takada T, Nishida K, Maeno K, Kakutani K, Yurube T, Doita M, et al. Intervertebral disc and macrophage interaction induces mechanical hyperalgesia and cytokine production in a herniated disc model in rats. Arthritis Rheum. (2012) 8:2601-2610.

17. VizcaínoRevés N, Mogel HM, Stoffel M, Summerfield A, Forterre F. Polarization of macrophages in epidural inflammation induced by canine intervertebral disc herniation. Front Vet Sci. (2020) 7:32.

18. Zhang H, Cai D, Bai X. Macrophages regulate the progression of osteoarthritis. Osteoarthritis Cartilage. (2020) 5:555-561.

19. Ma Y, Mouton AJ, Lindsey ML. Cardiac macrophage biology in the steady-state heart, the aging heart, and following myocardial infarction. Trans/ Res. (2018) 191:15-28. 20. Wang L, Zhang Y, Zhang N, Xia J, Zhan Q, Wang C. Potential role of $\mathrm{m} 2$ macrophage polarization in ventilator-induced lung fibrosis. Int immunopharmacol. (2019) 75:105795.

20. Zhang J, Muri J, Fitzgerald G, Gorski T, Gianni-Barrera R, Masschelein E, et al. Endothelial lactate controls muscle regeneration from ischemia by inducing $\mathrm{m} 2$-like macrophage polarization. Cell Metab. (2020) 6:1136-53.e1137.

21. Gensel JC, Zhang B. Macrophage activation and its role in repair and pathology after spinal cord injury. Brain Res. (2015) 1619:1-11.

22. Bogatcheva NV, Coleman ME. Conditioned medium of mesenchymal stromal cells: A new class of therapeutics. Biochemistry (Mosc). (2019) 11:1375-1389. 
23. Fernandes TL, Gomoll AH, Lattermann C, Hernandez AJ, Bueno DF, Amano MT. Macrophage: A potential target on cartilage regeneration. Front Immunol. (2020) 11:111.

24. Dai M, Sui B, Xue Y, Liu X, Sun J. Cartilage repair in degenerative osteoarthritis mediated by squid type ii collagen via immunomodulating activation of $\mathrm{m} 2$ macrophages, inhibiting apoptosis and hypertrophy of chondrocytes. Biomaterials. (2018) 180:91-103.

25. Zhang $\mathrm{H}$, Lin $\mathrm{C}$, Zeng $\mathrm{C}$, Wang $\mathrm{Z}$, Wang $\mathrm{H}$, Lu J, et al. Synovial macrophage m1 polarisation exacerbates experimental osteoarthritis partially through r-spondin-2. Ann Rheum Dis. (2018) 10:1524-1534.

26. Monchaux M, Forterre S, Spreng D, Karol A, Forterre F, Wuertz-Kozak K. Inflammatory processes associated with canine intervertebral disc herniation. Front Immunol. (2017) 8:1681.

27. Li X-C, Wang M-S, Liu W, Zhong C-F, Deng G-B, Luo S-J, et al. Co-culturing nucleus pulposus mesenchymal stem cells with notochordal cell-rich nucleus pulposus explants attenuates tumor necrosis factor-a-induced senescence. Stem Cell Res Ther. (2018) 1:171.

28. Johnson ZI, SchoepflinZR, Choi H, Shapiro IM, Risbud MV. Disc in flames: Roles of tnf- $a$ and il-1 $\beta$ in intervertebral disc degeneration. Eur Cell Mater. (2015) 30:104-116.

29. Bai XD, Li XC, Chen JH, Guo ZM, Hou LS, Wang DL, et al. Coculture with partial digestion notochordal cell-rich nucleus pulposus tissue activates degenerative human nucleus pulposus cells. Tissue Eng Part A. (2017) 15-16:837-846.

30. Ni L, Zheng Y, Gong T, Xiu C, Li K, Saijilafu, et al. Proinflammatory macrophages promote degenerative phenotypes in rat nucleus pulpous cells partly through erk and jnk signaling. $J$ Cell Physiol. (2019) 5:5362-5371.

31. Wang $Y$, Chang $T, W u T, X u$ W, Dou G, Wang $Y$, et al. M2 macrophages promote vasculogenesis during retinal neovascularization by regulating bone marrow-derived cells via sdf-1/vegf. Cell Tissue Res. (2020) 3:469-486.

32. Vizcaino Reves N, Mogel HM, Stoffel M, Summerfield A, Forterre F. Polarization of macrophages in epidural inflammation induced by canine intervertebral disc herniation. Front Vet Sci. (2020) 7:32.

33. Yao RR, Li JH, Zhang R, Chen RX, Wang YH. M2-polarized tumor-associated macrophages facilitated migration and epithelial-mesenchymal transition of hcc cells via the tlr4/stat3 signaling pathway. World JSurgOncol.(2018) 1:9.

34. Park HC, Quan H, Zhu T, Kim Y, Kim B, Yang HC. The effects of $m 1$ and $m 2$ macrophages on odontogenic differentiation of human dental pulp cells. J Endod. (2017) 4:596-601.

35. Lee S, Millecamps M, Foster DZ, Stone LS. Long-term histological analysis of innervation and macrophage infiltration in a mouse model of intervertebral disc injury-induced low back pain. JOrthopRes. (2020) 6:1238-1247.

36. Silva AJ, Ferreira JR, Cunha C, Corte-Real JV, Bessa-Gonçalves M, Barbosa MA, et al. Macrophages down-regulate gene expression of intervertebral disc degenerative markers under a pro-inflammatory microenvironment. Front Immunol. (2019) 10:1508. 
37. Choi H, Tessier S, Silagi ES, Kyada R, Yousefi F, Pleshko N, et al. A novel mouse model of intervertebral disc degeneration shows altered cell fate and matrix homeostasis. Matrix Biol. (2018) 70:102-122.

38. Wang R, Xu B, Xu H. Tgf- $\beta 1$ promoted chondrocyte proliferation by regulating sp1 through mscexosomes derived mir-135b. Cell Cycle. (2018) 24:2756-2765.

39. Liu J, Li SM. Mir-484 suppressed proliferation, migration, invasion and induced apoptosis of gastric cancer via targeting ccl-18. Int J Exp Pathol. (2020) 6:203-214.

40. Qiu X, Zhuang M, Lu Z, Liu Z, Cheng D, Zhu C, et al. Ripk1 suppresses apoptosis mediated by tnf and caspase-3 in intervertebral discs. J Trans/ Med. (2019) 1:135. doi: 10.1186/s12967-019-1886-3.

41. Ding F, Shao ZW, XiongLM. Cell death in intervertebral disc degeneration. Apoptosis. (2013) 7:777785.

42. Wang F, Cai F, Shi R, Wang XH, Wu XT. Aging and age related stresses: A senescence mechanism of intervertebral disc degeneration. Osteoarthritis Cartilage. (2016) 3:398-408.

43. Wang WJ, Yu XH, Wang C, Yang W, He WS, Zhang SJ, et al. Mmps and adamtss in intervertebral disc degeneration. Clin ChimActa. (2015) 448:238-246.

44. Feng $C$, Liu H, Yang M, Zhang Y, Huang B, Zhou Y. Disc cell senescence in intervertebral disc degeneration: Causes and molecular pathways. Cell Cycle. (2016) 13:1674-1684.

45. Dimri GP, Lee X, Basile G, Acosta M, Scott G, Roskelley C, et al. A biomarker that identifies senescent human cells in culture and in aging skin in vivo. Proc NatiAcad Sci U S A. (1995) 20:9363-9367.

46. Gantenbein B, Illien-Jünger S, Chan SC, Walser J, Haglund L, Ferguson SJ, et al. Organ culture bioreactors-platforms to study human intervertebral disc degeneration and regenerative therapy. CurrStem Cell Res Ther. (2015) 4:339-352.

47. Ji ML, Jiang H, Zhang XJ, Shi PL, Li C, Wu H, et al. Preclinical development of a microrna-based therapy for intervertebral disc degeneration. Nat Commun. (2018) 1:5051.

48. Tam V, Chan WCW, Leung VYL, Cheah KSE, Cheung KMC, Sakai D, et al. Histological and reference system for the analysis of mouse intervertebral disc. JOrthopRes. (2018) 1: 233-243.

49. Mills CD. M1 and m2 macrophages: Oracles of health and disease. CritRev Immunol. (2012) 6: 463488.

\section{Tables}




\begin{tabular}{|llllll|}
\hline \multicolumn{5}{|c|}{ Table 1: Detailed characteristics of the enrolled patients } \\
\hline Case No. & Sex & Age(years) & Diagnosis & Disc level & Pfirrmann grade \\
\hline Case 1 & Male & 20 & LDH & L4-L5 & III \\
\hline Case 2 & Male & 26 & LDH & L4-L5 & III \\
\hline Case 3 & Male & 39 & LDH & L5-S1 & III \\
\hline Case 4 & Male & 27 & LDH & L4-L5 & III \\
\hline Case 5 & Male & 31 & LDH & L4-L5 & III \\
\hline Case 6 & Male & 30 & LDH & L5-S1 & III \\
\hline Case 7 & Male & 42 & LDH & L5-S1 & III \\
\hline Case 8 & Male & 25 & LDH & L4-L5 & III \\
\hline LDH, lumbar disk herniation. & & & \\
\hline
\end{tabular}

Figures 
A

A

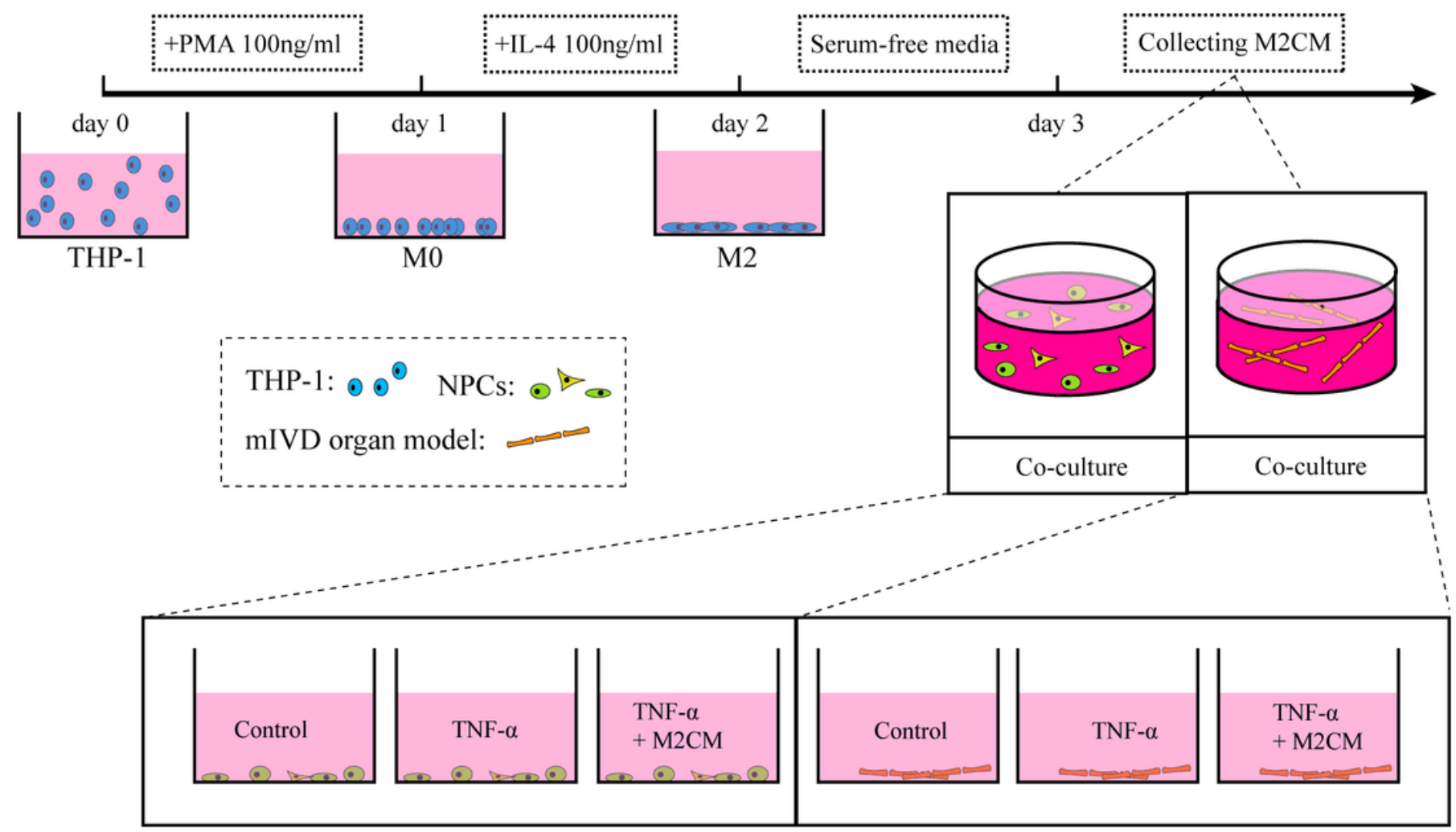

B

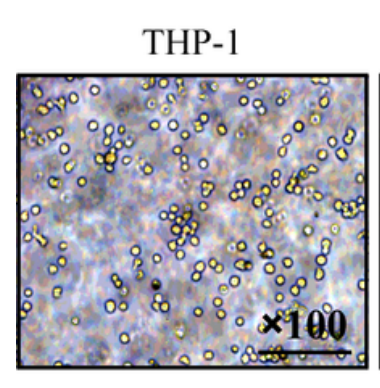

$\mathrm{C}$



$\mathrm{D}$

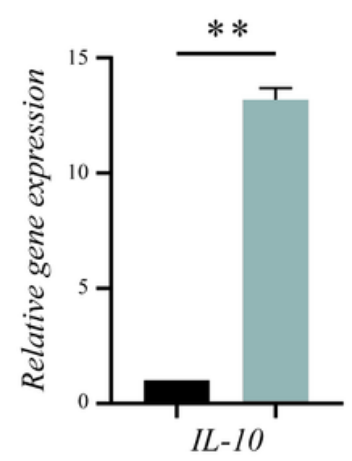

\section{Figure 1}

Flow diagram of harvest of M2 macrophage-conditioned medium (M2CM) and the identification of M2 macrophages. (A) The process of M2CM harvesting and grouping. (B)Morphology of THP-1 cells in different induction states. (C and D)Gene expression of M2 macrophage markers CD206 and IL-10 was significantly higher than that of $M 0$ macrophages, $n=3$ donors, $* * p<0.01$. 


\section{A}

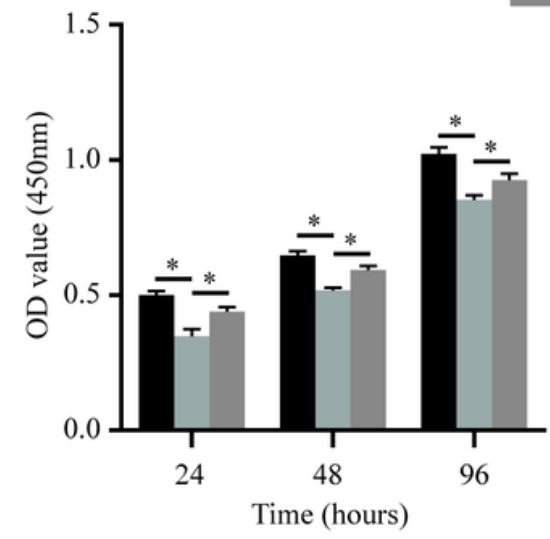

C

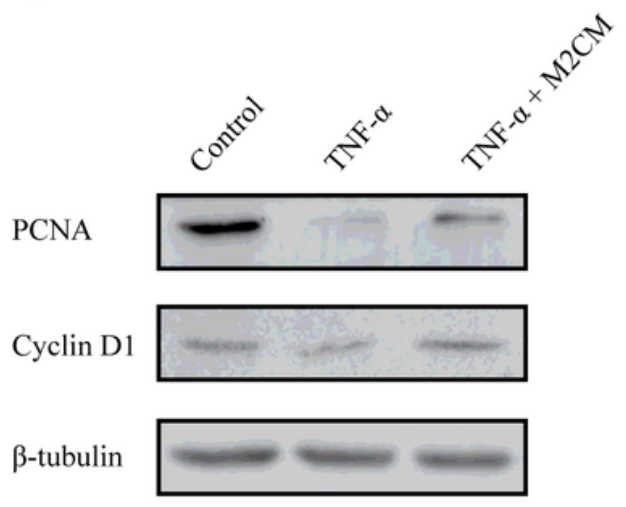

Control

TNF- $\alpha$

TNF- $\alpha+$ M 2 CM
B

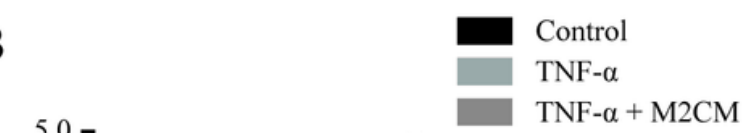

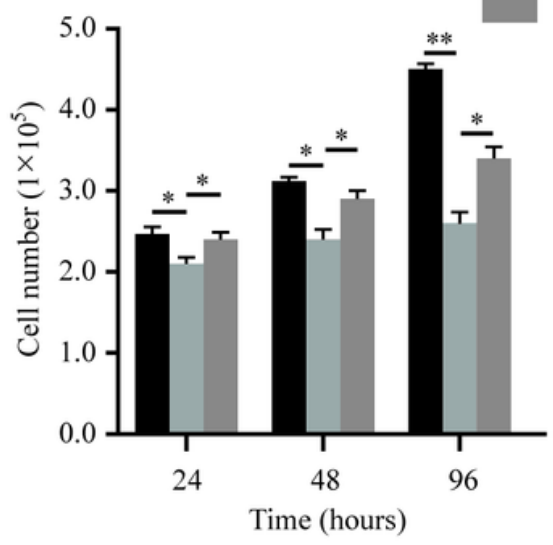

D

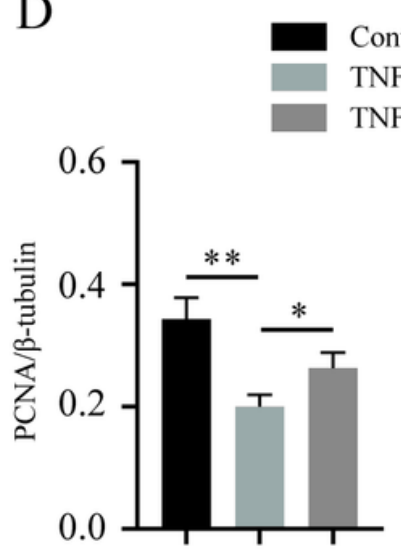

E

ontrol

NF- $\alpha$ Control TNF- $\alpha$ TNF- $\alpha+$ M 2 CM

Figure 2

Effect of macrophage-conditioned medium (M2CM) on the proliferation of TNF-a-treated NPCs. (A) Cell proliferation analysis of CCK-8 in the control, TNF-a, and TNF-a+M2CM groups at 24, 48, and 96h; n = 3 donors, ${ }^{*} p<0.05$. (B)The number of NPCs was similar, but significantly declined at 24,48 , and $96 \mathrm{~h}$; however, co-culturing with M2CM attenuated this trend. $n=3$ donors, ${ }^{*} p<0.05,{ }^{* *} p<0.01$. Western blot analysis (C) and densitometric quantification of PCNA (D) and cyclin D1 (E) proteins in the three groups. Data are expressed as mean $\pm S D ; n=3$ donors, $* p<0.05, * * p<0.01$. 

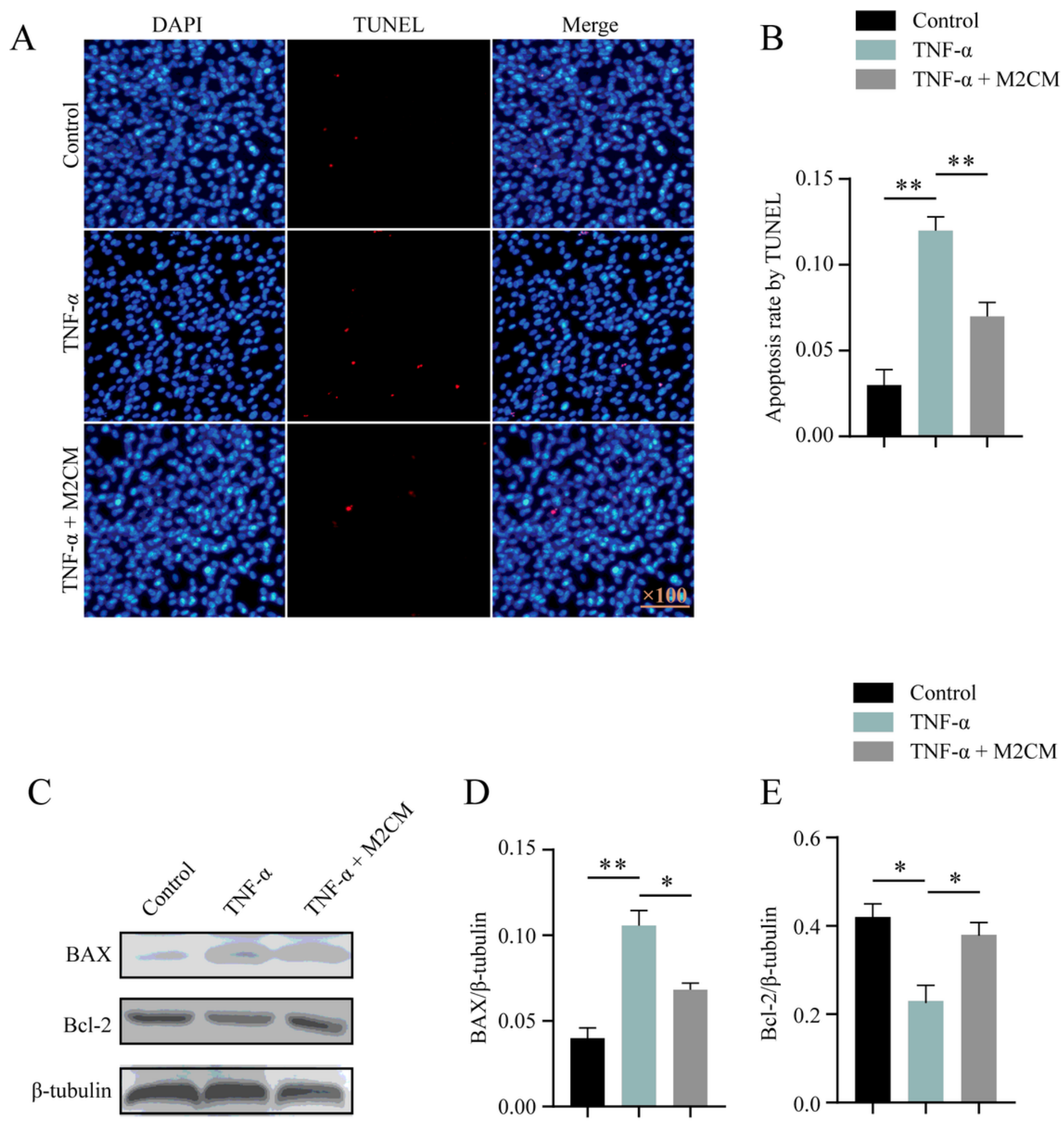

$\mathrm{E}$

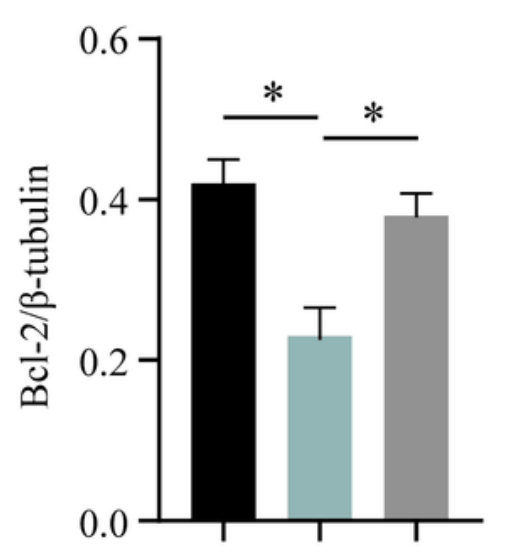

\section{Figure 3}

Effects of macrophage-conditioned medium (M2CM) on the apoptosis of TNF-a-treated NPCs. TUNEL staining $(A)$ and the change in the apoptotic rates $(B)$ in the control, TNF-a treated, and the co-culture groups; $n=3$ donors, ${ }^{* *} p<0.01$. Western blot analysis (C), and the protein levels of $B A X(D)$ and $B c l-2(E)$ were analyzed in the three groups. All data are expressed as mean $\pm S D ; n=3$ donors, ${ }^{*} p<0.05,{ }^{* \star} p<0.01$. 
A

Collage II

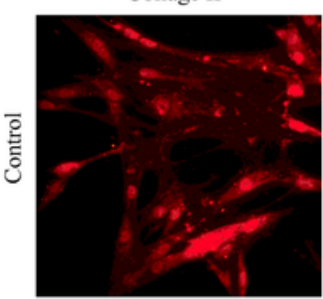

$\underset{b}{i}$
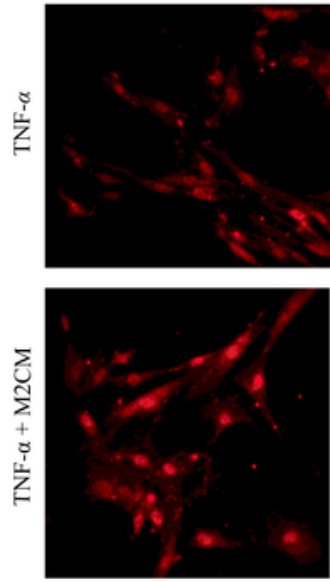

C

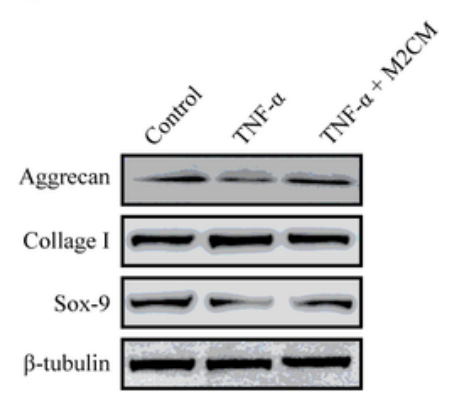

D
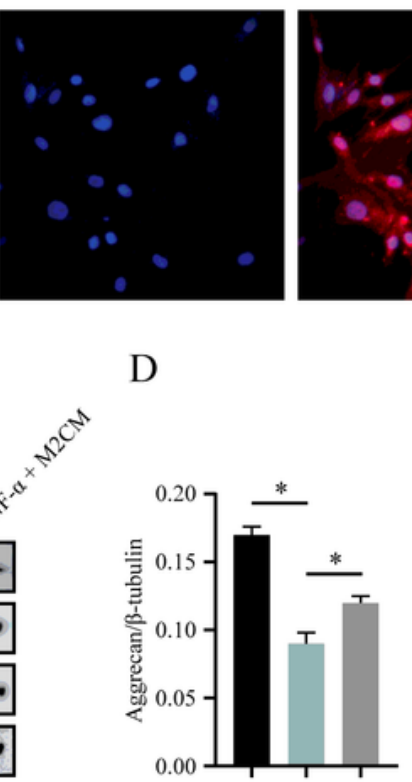
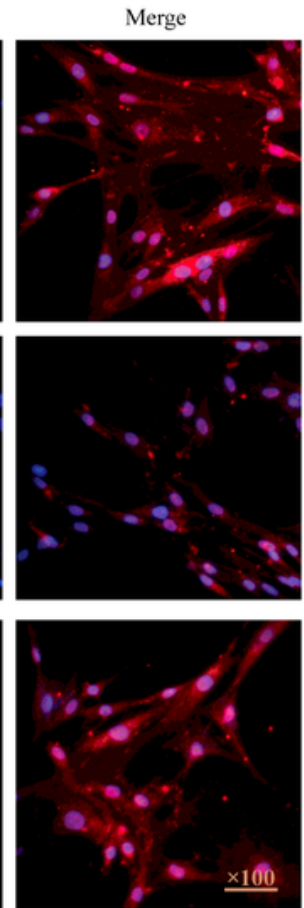

E
- Control

TNF- $\alpha$

TNF- $\alpha+$ M2CM

B

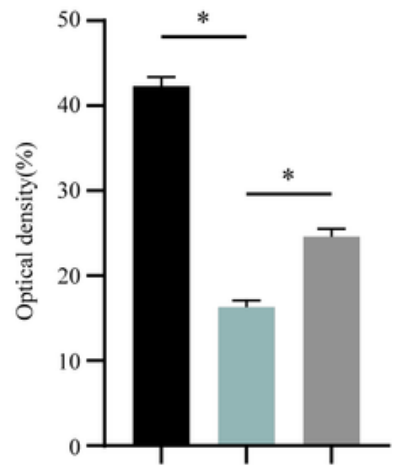

G

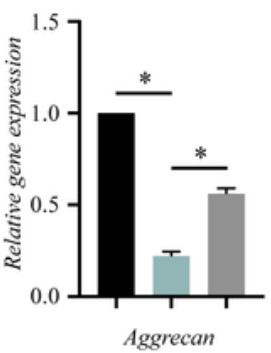

$\mathrm{H}$

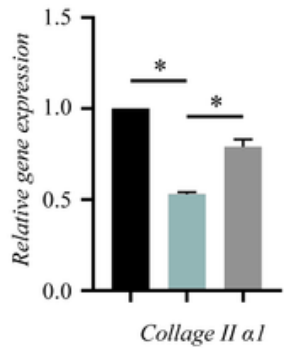

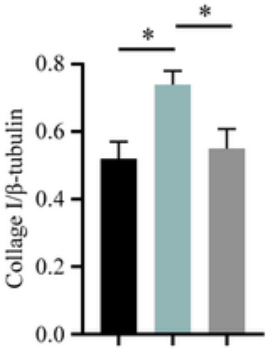

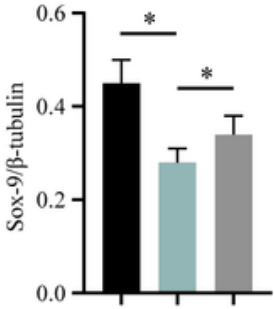

Figure 4

Effects of macrophage-conditioned medium (M2CM) on the expression of matrix macromolecules in TNF-a-treated NPCs. Representative images of immunofluorescence staining $(A)$ and quantification analysis of collagen II protein expression(B) in the three groups after 3 days of culture; $n=3$ donors, ${ }^{*} p<$ 0.05 , ${ }^{\star *} p<0.01$. Western blot analysis(C) and densitometric quantification of aggrecan (D) and collagen I (E ) proteins in the three groups; $\mathrm{n}=3$ donors, ${ }^{*} \mathrm{p}<0.05$. The mRNA expression levels of aggrecan $(F)$, 
collagen la1(G), and collage lla1(H) in NPCs were compared among different groups after 3 days of culture. All data are expressed as mean $\pm S D, n=3$ donors, * $p<0.05$, ** $p<0.01$, *** $p<0.001$.
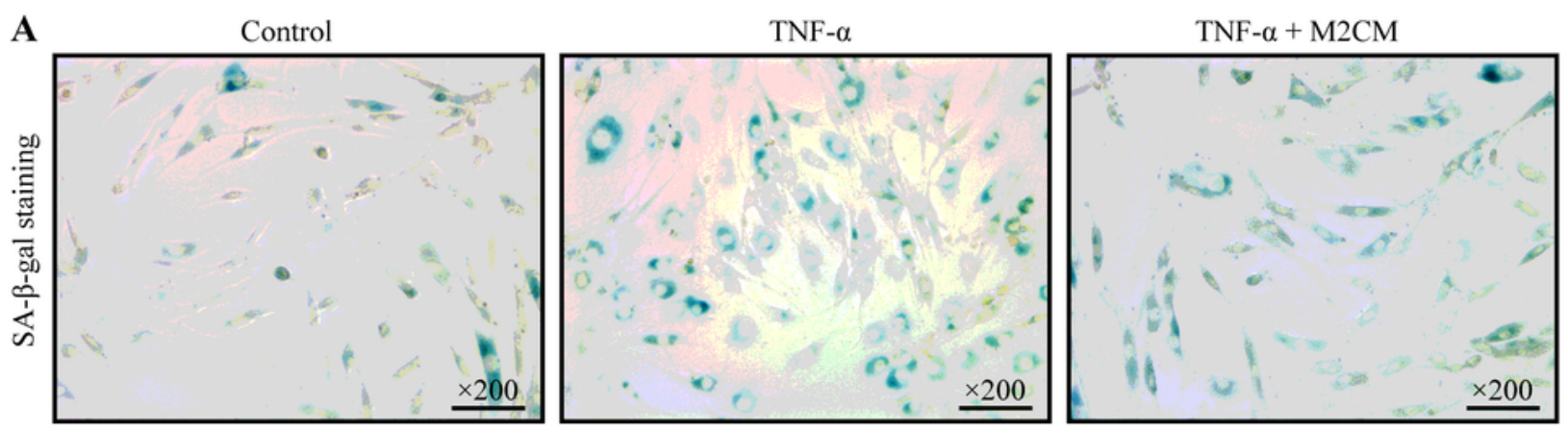

Control

TNF- $\alpha$

$\mathrm{TNF}-\alpha+\mathrm{M} 2 \mathrm{CM}$

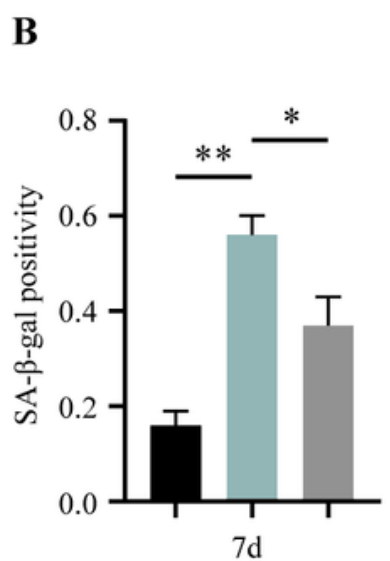

C
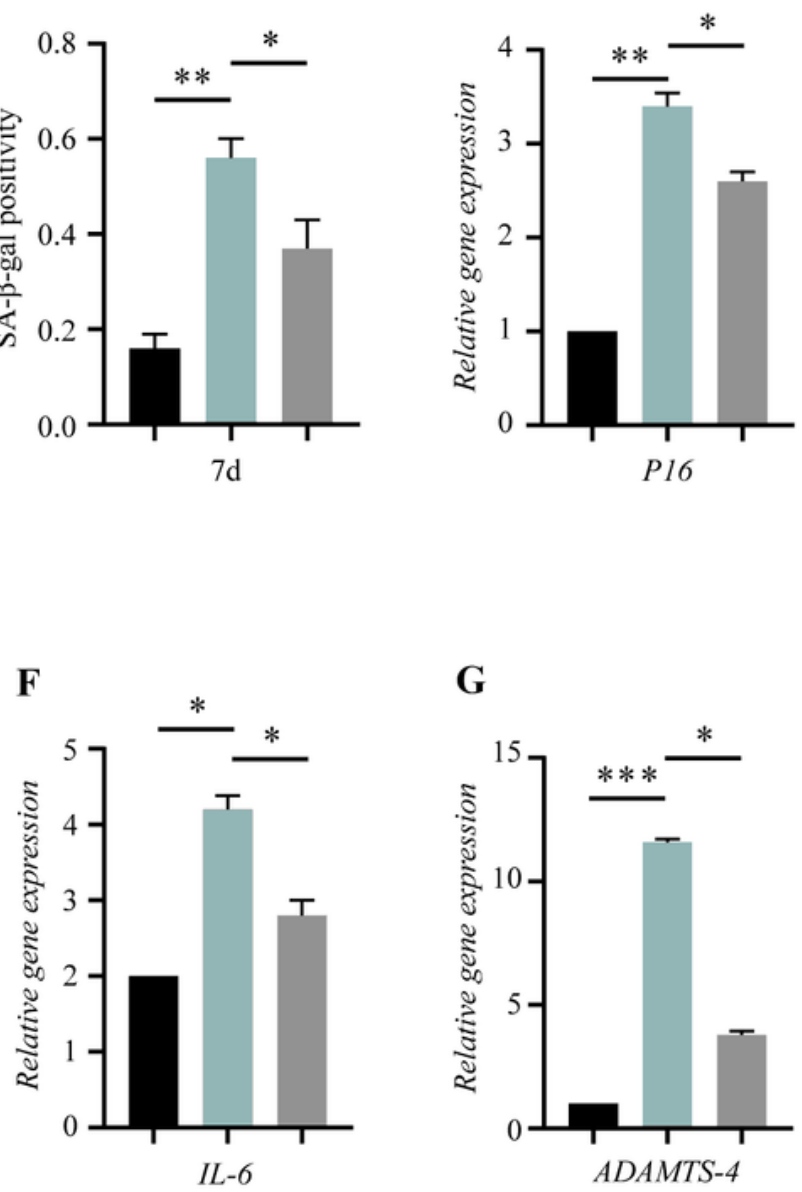

G

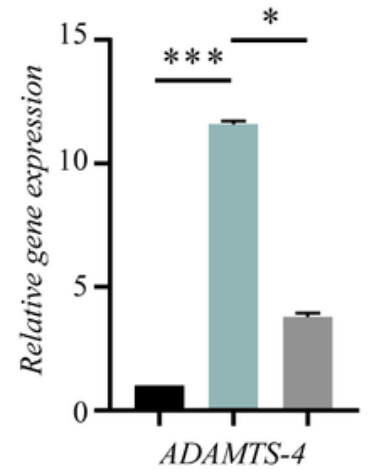

D

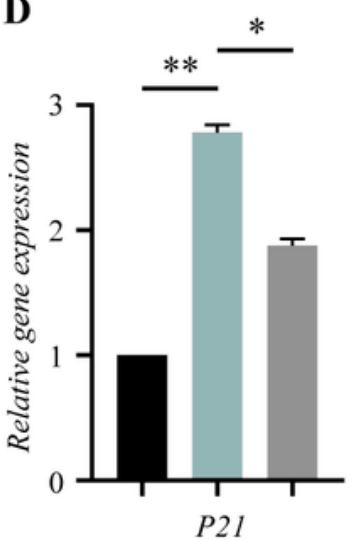

H

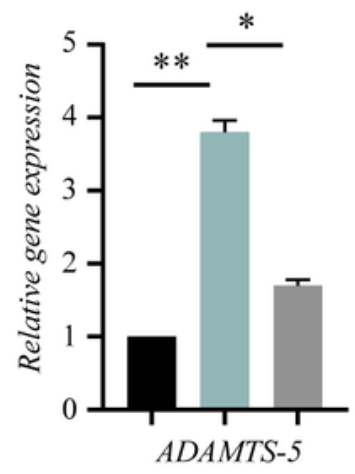

$\mathbf{E}$

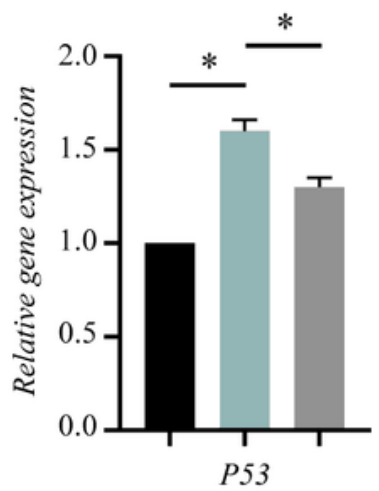

Control TNF- $\alpha$ TNF- $\alpha+$ M 2 CM

I

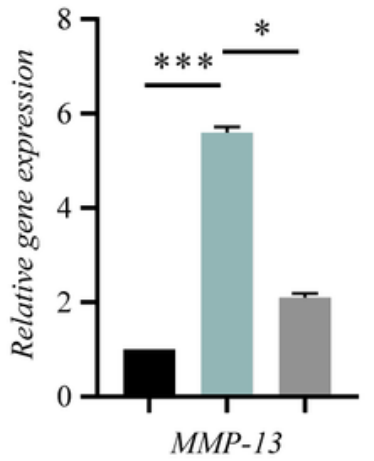

Figure 5

Effects of macrophage-conditioned medium (M2CM) on cell senescence and inflammation factors in TNF-a-treated NPCs. Cell senescence-associated $\beta$-galactosidase (SA- $\beta$-gal) staining after 7 days of culture (A). Positive staining of SA- $\beta$-gal was compared among different groups (B);n $=3$ donors, * $p<$ 
$0.05, * \star p<0.01$. Real-time PCR analysis of p16 (C), p21 (D), and p53 (E) mRNA expression; the inflammation factors (F: IL-6) and matrix degrading enzymes(G: ADAMTS-4, H: ADAMTS-5, I: MMP-13) in TNF-a-induced NPCs treated with and without M2CM were analyzed. Data are expressed as mean \pm SD values, $n=3, * p<0.05, * * p<0.01, * \star * p<0.001$.

A
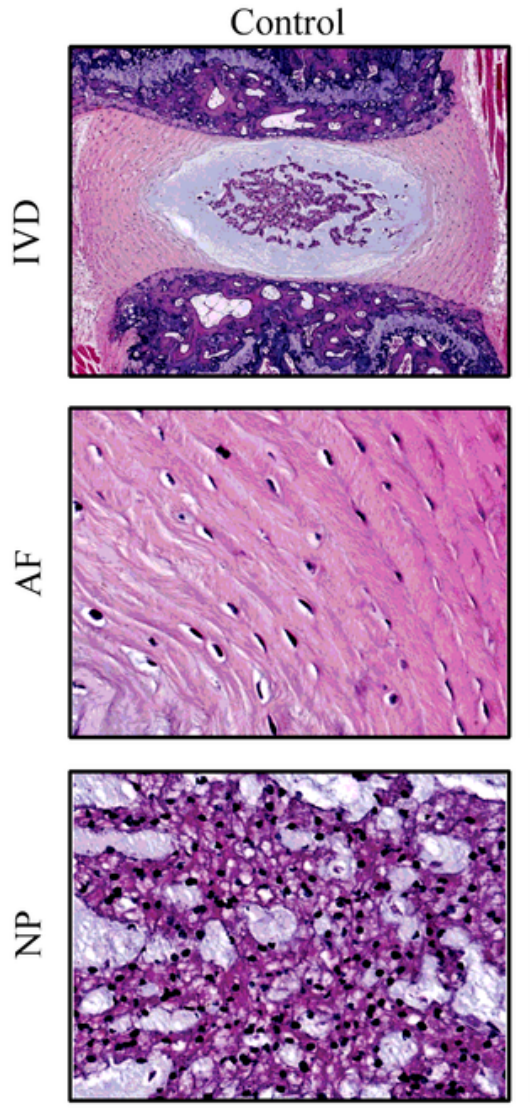

TNF- $\alpha$
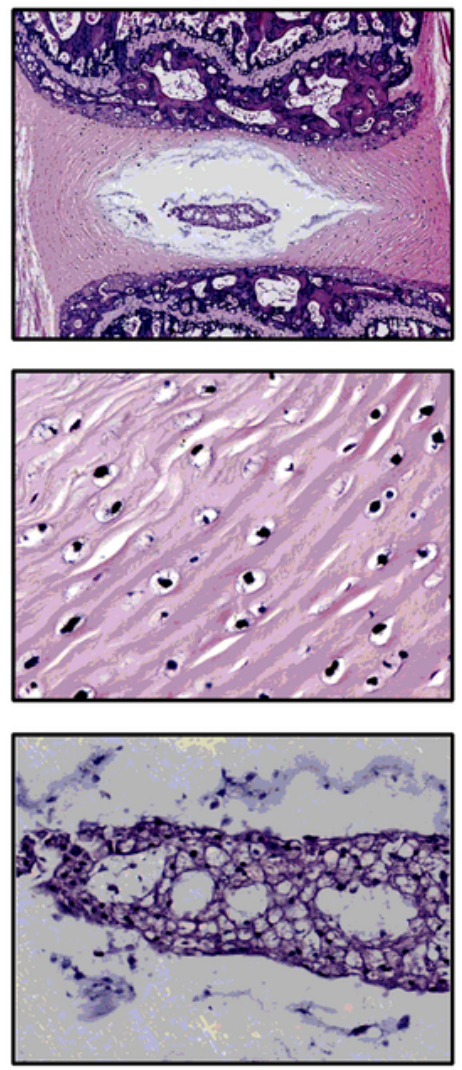

TNF- $\alpha+\mathrm{M} 2 \mathrm{CM}$


Control

TNF- $\alpha$

TNF- $\alpha+\mathrm{M} 2 \mathrm{CM}$
B

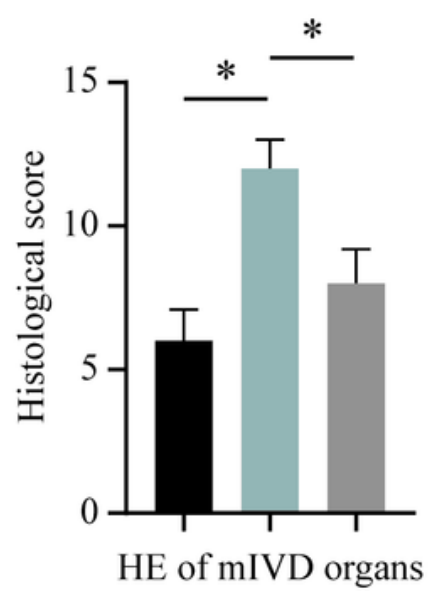

C

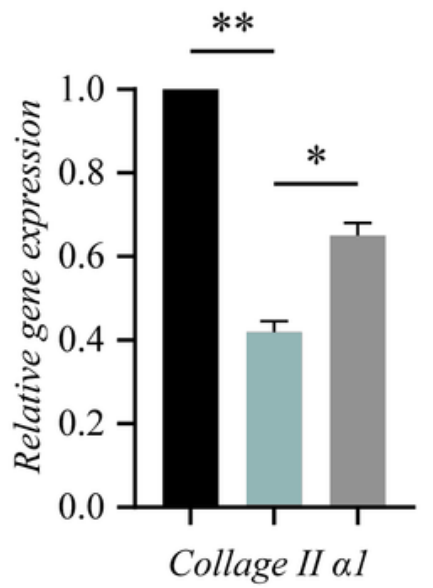

D

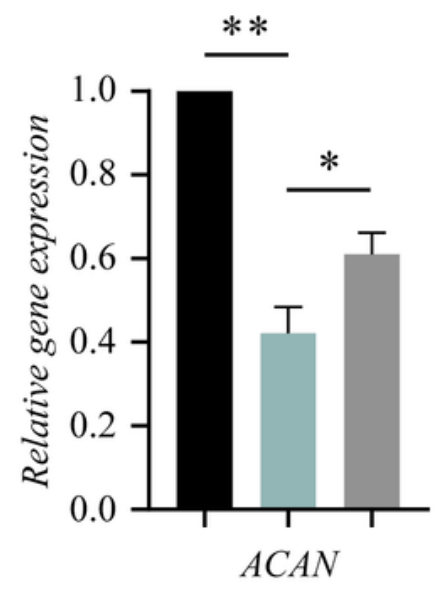

Figure 6 
Effects of macrophage-conditioned medium (M2CM) on mouse intervertebral discs (mIVDs and the gene expression of collagen lla1 and aggrecan. Representative image of HE staining of mIVDs (A) and quantification analysis histological scores (B); $n=5, * p<0.05$. The mRNA expression levels of collagen lla1 (C) and aggrecan (D) in mIVDs were compared among different groups after 14 days of culture. All data are expressed as mean $\pm S D ; n=5, * p<0.05$, ** $p<0.01$.

\section{Supplementary Files}

This is a list of supplementary files associated with this preprint. Click to download.

- TableS1.docx 